\title{
Clinical anxiety, cortisol and interleukin-6: Evidence for specificity in emotion-biology relationships
}

\author{
Aoife O'Donovan ${ }^{a, b,{ }^{*}, \text { Brian M. Hughes }}{ }^{c}$, George M. Slavich $^{b}$, Lydia Lynch ${ }^{d}$, Marie-Therese \\ Cronin $^{e}$, Cliona O'Farrelly ${ }^{f}$, and Kevin M. Malone ${ }^{a}$
}

aDepartment of Psychiatry and Mental Health Research, St. Vincent's University Hospital and School of Medicine and Medical Sciences, University College Dublin, Ireland bepartment of Psychiatry, University of California, San Francisco, USA ${ }^{\circ}$ Centre for Research on Occupational and Life Stress, National University of Ireland, Galway, Ireland dEducation and Research Centre, St. Vincent's University Hospital, Dublin, Ireland eSt. Vincent's University Hospital, Dublin, Ireland fSchool of Biochemistry and Immunology, Trinity College Dublin, Ireland

\begin{abstract}
Anxiety confers increased risk for inflammatory diseases, and elevated inflammatory activity in anxious individuals may contribute to this increased risk. One complication, however, is that anxiety could be associated with inflammatory activity either through a specific anxiety pathway or through a more general negative emotionality pathway. To investigate, we measured levels of the stress hormone cortisol, the pro-inflammatory cytokine interleukin-6 (IL-6), and the systemic inflammatory marker C-reactive protein (CRP), as well as depression and neuroticism, in clinically anxious and non-anxious adults. Compared with non-anxious participants, clinically anxious participants exhibited significantly lower levels of morning cortisol and significantly higher levels of IL-6, independent of age, sex, and depressive symptoms. These group differences were robust when controlling for neuroticism. Conversely, the groups had equivalent levels of CRP in all analyses. Results are indicative of anxiety-specific effects on inflammatory activity, and highlight a pathway by which anxiety may increase risk for inflammatory diseases.
\end{abstract}

\section{Keywords}

Anxiety; Depression; Cortisol; Interleukin-6; Neuroticism

\section{Introduction}

\begin{abstract}
Negative emotions such as anxiety and depression confer increased risk for disorders with an inflammatory etiology, and elevated inflammatory activity may be an important mediator of emotion-disease relationships (Kiecolt-Glaser et al., 2002). However, whereas a large number of studies have examined associations between depression and inflammation
\end{abstract}

(C) 2010 Elsevier Inc. All rights reserved

*Corresponding author at: Department of Psychiatry, University of California, San Francisco, USA. aoife.odonovan@ucsf.edu (Aoife O'Donovan). 
(Dantzer et al., 2008; Whooley et al., 2007), little is known about the relationship between anxiety and inflammation. The paucity of research on this topic is particularly striking because anxiety may be an even stronger risk factor for inflammatory disorders than depression (Kubzansky and Kawachi, 2000; Roy-Byrne et al., 2008).

Specific cognitive biases towards threat-related information may play a key role in the relationship between anxiety and inflammation (Mogg and Bradley, 2005). A growing body of research indicates that exposure to real or imagined psychological threats activates multiple biological systems, including the hypothalamic-pituitary adrenal (HPA) axis, which regulates inflammatory activity, and the inflammatory response (Slavich et al., in press). Exaggerated and sustained threat perception in anxious individuals may result in chronic activation of the biological stress response, leading to reduced secretion of the HPA axis hormone cortisol during the morning period (Miller et al., 2007), and elevated inflammatory activity (Kiecolt-Glaser et al., 2003).

Early research examining the effects of psychological states on biological systems was based on a generality model, derived from the observation that various physical and psychological stimuli elicit a common pattern of biological reactions (Selye, 1956). However, more recently, an integrated specificity model has been proposed, emphasizing that specific psychological states are associated with specific patterns of biological responses (Kemeny, 2003; Moons et al., 2010). Anxiety and depression are highly comorbid with one another, and both emotions overlap with neuroticism, or the general tendency to experience negative emotions (Jylhä and Isometsä, 2006). As such, any observed associations between anxiety and inflammation could be due to general negative emotionality or specific features of anxiety. However, anxiety appears to confer increased risk for CVD independent of associations with depression (Kubzansky and Kawachi, 2000), threat-related attentional biases are not observed in depression (Mogg and Bradley, 2005). Although neuroticism has been associated with exaggerated threat perception (Bishop, 2008), evidence for associations between neuroticism and inflammation is mixed (Pitsavos et al., 2006; Toker et al., 2005). Thus, we hypothesize that clinically anxious participants will have lower morning cortisol and higher levels of inflammatory markers compared with non-anxious participants, independent of comorbid depressive symptoms and neuroticism.

\section{Methods}

\subsection{Participants}

Participants were recruited via posters, flyers and emails distributed in a large suburban area in Dublin, Ireland. Promotional materials stated that the study was recruiting individuals currently experiencing high or low levels of psychological distress, and that participants would receive a $€ 10$ book token. The sample included 27 participants with Hospital Anxiety and Depression Scale Anxiety (HADS-A) scores in the clinical range (anxious group [scores 28]: $M$ HADS-A $=10.04, S D=1.91 ; M$ age $=33.52, S D=8.04)$ and 29 participants with low scores on the HADS-A (non-anxious group [scores < 8]: $M$ HADS-A = 5.17, $S D=1.49$; $M$ age $=30.97, S D=10.21$; Zigmond and Snaith, 1983). All participants were white and $68 \%$ were female. Exclusion criteria included the presence of chronic illness, acute illness within the previous two weeks, possible current infection, alcoholism, use of medication 
(apart from the oral contraceptive pill), anesthesia in the previous three months, and night shift work in the previous two weeks. The study was approved by the local institutional ethics committee.

\subsection{Materials and measures}

2.2.1. Anxiety and depression-The Hospital Anxiety and Depression Scale (HADS) is a widely used and well-validated measure that assesses non-somatic symptoms of anxiety and depression (Zigmond and Snaith, 1983). The HADS includes a 7-item anxiety scale (HADS-A) and a 7-item depression scale (HADS-D). A review of 747 studies confirmed the validity of the HADS in assessing severity of anxiety and depression, and determined that the HADS has sensitivity and specificity of approximately $80 \%$ in defining caseness for anxiety and depressive disorders based on a clinical cutoff score of 8 (Bjelland et al., 2002; Crawford et al., 2001). Internal consistency for the HADS-A $(a=.72)$ and HADS-D $(a=$. 63) were acceptable.

2.2.2. Neuroticism-Neuroticism was assessed with the 12-item Eysenck Personality Questionnaire-Revised, Short Form, which has reliability and validity comparable with the full-length EPQ (Barrett and Eysenck, 1992). Internal consistency for the neuroticism scale was acceptable $(\alpha=.80)$.

2.2.3. Health status—Self-reported physical health measures included: self-ratings of physical health "right now" as "excellent", "very good", "good", "fair" or "poor"; acute illness (e.g., cold or influenza) during the previous three months (cold/flu incidence); and past and current chronic illnesses.

2.2.4. Cortisol-Salivettes were used to obtain saliva samples (Sartstedt Inc., Germany). Each salivette comprised a cotton dental roll contained within two sterile plastic tubes. Participants were instructed to place the cotton roll in their mouth for a minimum of $90 \mathrm{~s}$ to saturate it with saliva before returning it to the plastic tube, which was then capped tightly and stored at $-20{ }^{\circ} \mathrm{C}$. When all samples were ready for analysis, tubes were centrifuged for $10 \mathrm{~min}$ at 3000RPM for separation of supernatant. Salivary cortisol analyses were performed in duplicate using a commercially available high sensitivity salivary cortisol enzyme immunoassay (Salimetrics LLC, USA). Intra- and inter-assay coefficients of variation were $<7 \%$.

2.2.5. IL-6 and hsCRP-Blood was collected in clot activator vacutainer tubes (Becton, Dickinson \& Company, USA) for the measurement of IL-6 and high sensitivity CRP (hsCRP). Serum samples were obtained following centrifugation at 2000RPM for $10 \mathrm{~min}$, and stored at $-20^{\circ} \mathrm{C}$ until analysis. Analyses for IL-6 were performed in duplicate using a commercially available high sensitivity IL-6 ELISA (R\&D Systems, USA). Intra- and interassay coefficients of variation were $<10 \%$. High sensitivity tests of CRP permit the examination of levels within the low-normal range (hsCRP, Ridker, 2001). Analyses for hsCRP were performed by means of particle enhanced immunonephelometry using CardioPhase hsCRP reagents on the BN System (Dade Behring, USA). The intra-assay coefficient of variation for hsCRP was $<6 \%$, and all samples were analysed on a single run. 
Due to insufficient availability of serum for four participants, CRP results are based on 52 participants.

\subsection{Procedure}

Biological samples were collected between 7:30 and 9:30AM. Participants fasted and abstained from smoking and caffeine from midnight, and avoided alcohol and exercise for $24 \mathrm{~h}$ prior to their appointment. Females were scheduled to participate during the follicular phase of the menstrual cycle. Participants were instructed to avoid rushing if they were late, and travel expenses were awarded so that they would not walk or bike to their appointment. Upon arrival at the laboratory, participants gave written informed consent and then completed a screener questionnaire. This process lasted approximately $10 \mathrm{~min}$, during which time participants were at rest. Following this rest period, participants provided a saliva sample and then a $15 \mathrm{ml}$ venous blood sample was taken from the antecubital fossa area. Finally, participants completed questionnaires under the supervision of the study coordinator.

\subsection{Data analysis}

Pearson's correlations were used to assess relationships between continuous variables. Differences between anxious and non-anxious participants in cortisol, IL-6 and hsCRP were assessed with Student's $t$-tests. To test the hypothesis that between-group differences were independent of potential covariates, we reran our primary model controlling for age, gender, depression and neuroticism using analysis of covariance.

\section{Results}

\subsection{Anxiety and inflammation}

All participants were in good physical health as determined by self-report. Self-reported physical health was not associated with cortisol, IL-6, or hsCRP levels. Differences between anxious and non-anxious participants in study variables and potential confounds are presented in Table 1. While cortisol was not significantly associated with either of the inflammatory markers, IL-6 and hsCRP were significantly associated with one another $(r=$. $32, p=.03)$. Correlational analyses indicated that higher levels of anxiety were marginally related to higher levels of IL-6 $(r=.27, p=.05)$ and lower levels of cortisol $(r=.25, p=$. 06), but not with levels of hsCRP (Fig. 1a and b). Depression, on the other hand, was not associated with any of the biological variables.

Compared to non-anxious participants, clinically anxious participants had significantly lower levels of cortisol, $t(54)=2.84, p=.006$, and significantly higher levels of IL-6, $t(50)$ $=2.01, p=.05$ (Fig. 1a and b), but they did not differ in levels of hsCRP, $t(49)=0.15, p=$. 88.

As predicted, levels of anxiety and depression were strongly correlated $(r=.51, p<.001)$. Given that depression has previously been associated with cortisol and IL-6 levels, and that anxious participants exhibited significantly higher levels of depression than non-anxious participants, $t(54)=2.28, p=.03$, it is possible that depression contributed to the observed 
differences in levels of cortisol and IL-6 between anxious and non-anxious participants.

However, when depression was added as a covariate in our model together with age and sex, clinically anxious participants continued to exhibit significantly lower levels of cortisol, $F(1,56)=6.05, p=.02$, partial $\eta^{2}=.11$, as well as higher levels of IL-6, $F(1,52)=4.16, p$ $=.047$, partial $\eta^{2}=.08$. Although anxious participants did not have significantly higher levels of neuroticism than non-anxious participants, $t(48)=1.42, p=.16$, raw anxiety and neuroticism scores were significantly associated $(r=.28, p=.04)$. When we included neuroticism as an additional covariate, the effect size for the between-group difference in cortisol remained similar (even though the finding became marginally non-significant), $F(1,44)=3.82, p=.06$, partial $\eta^{2}=.08$, and clinically anxious participants continued to exhibit significantly higher levels of IL-6, $F(1,40)=4.31, p=.04$, partial $\eta^{2}=.10$.

\section{Discussion}

Our data indicate that clinically anxious individuals have lower levels of morning cortisol and higher levels of the pro-inflammatory cytokine IL-6. The difference between anxious and non-anxious participants in cortisol and IL-6 was present even when controlling for both depression and neuroticism, indicating some degree of specificity in the relationship between anxiety and IL-6. We found no difference between anxious and non-anxious participants in levels of CRP, another inflammatory marker. There are a number of possible reasons for our finding that anxious participants differed from non-anxious participants on one marker of inflammation but not another. Notably, the psychosomatic pathway that determines stress-related production of IL-6 is likely to differ in important ways from that leading to production of CRP. For example, previous research suggests that, unlike CRP, IL-6 may be subject to direct influence by neural and hormonal responses to stress (Zhou et al., 1993). Overall, these data provide support for the notion of specificity at psychological and biological levels in the relation between negative emotional states and inflammation (Kemeny, 2003).

Cognitive biases towards threatening information may lead to exaggerated threat perception and consequently, more frequent and prolonged activation of the biological stress response in anx ious individuals. Given that anxiety disorders tend to have an early onset and a chronic course (Kessler et al., 2005; Thyer et al., 1985), exaggerated threat perception across many years could manifest as chronic exposure to biological stress reactivity. In fact, the pattern of lower morning cortisol and elevated IL-6 that anxious participants exhibited in the present study resembles the pattern previously observed in association with chronic stress (Kiecolt-Glaser et al., 2003; Miller et al., 2007). Further research is necessary to examine if chronic exaggerated threat perception mediates this effect.

In contrast with the large body of research examining the association between depression and inflammation (Dantzer et al., 2008), evidence regarding the association between anxiety and inflammatory activity is limited. Furthermore, the limited research on state anxiety and inflammation has yielded a mixed pattern of results (Chandrashekara et al., 2007; Maes et al., 1998). Different degrees of depression and neuroticism within anxious individuals, differences in severity of anxious symptoms, and variation in specific inflammatory proteins measured across studies could account for this mixed pattern of findings. The present data 
highlight that it is inappropriate to hypothesize simple emotion-inflammation relationships.

Rather, investigators must pay attention to characteristics of the specific negative emotion of interest, the level at which that emotion is experienced, and to the complex network of proteins involved in the inflammatory response.

\subsection{Limitations and future directions}

The present findings must be interpreted in light of several limitations. First, although our protocol involved collecting all biological samples during a two-hour period, it is possible that differences between anxious and non-anxious participants in sleep disruption or time since awakening contributed to our findings. Second, the cross-sectional design of the present research does not permit us to determine causal direction in the relations observed between anxiety, cortisol, and IL-6. However, pro-inflammatory cytokines appear to selectively promote symptoms of depression without influencing levels of anxiety (Capuron et al., 2001; Lieb et al., 2006), supporting the formulation that the direction of effect would be from anxiety to inflammation and not vice versa. Finally, the present small study was a first step towards examining anxiety-specific effects on inflammatory activity. IL-6 is an important target for psychoneuroimmunological study because of the key role this cytokine plays in regulating normal inflammatory responses and in the pathophysiology of numerous inflammatory diseases. However, a more complete understanding of anxiety effects on specific aspects of the inflammatory response would require the measurement of multiple pro- and anti-inflammatory cytokines.

\section{Conclusions}

In sum, the present data indicate that clinically anxious individuals have lower morning cortisol and elevated IL-6 compared with non-anxious individuals, highlighting a potential pathway by which anxiety may increase risk for inflammatory diseases. Importantly, our findings suggest that anxiety may be associated with inflammatory activity independent of depression and neuroticism, thus indicating specificity in relationships between negative emotions and biological responses. Consequently, they support the adoption of an integrated specificity model to elucidate relationships between negative emotions and inflammation.

\section{References}

Barrett PT, Eysenck SB. Predicting EPQ-R full scale scores from the short form version. Pers. Indiv. Differ. 1992; 13:851-853.

Bishop SJ. Neural mechanisms underlying selective attention to threat. Ann. NY Acad. Sci. 2008; 1129:141-152. [PubMed: 18591476]

Bjelland I, Dahl AA, Haug TT, Neckelmann D. The validity of the Hospital Anxiety and Depression Scale. An updated literature review. J. Psychosom. Res. 2002; 52:69-77. [PubMed: 11832252]

Capuron L, Ravaud A, Gualde N, Bosmans E, Dantzer R, Maes M, Neveu PJ. Association between immune activation and early depressive symptoms in cancer patients treated with interleukin-2based therapy. Psychoneuroendocrinology. 2001; 26:797-808. [PubMed: 11585680]

Chandrashekara S, Jayashree K, Veeranna HB, Vadiraj HS, Ramesh MN, Shobha A, Sarvanan Y, Vikram YK. Effects of anxiety on TNF-alpha levels during psychological stress. J. Psychosom. Res. 2007; 63:65-69. [PubMed: 17586339]

Crawford JR, Henry JD, Crombie C, Taylor EP. Normative data for the HADS from a large nonclinical sample. Br. J. Clin. Psychol. 2001; 40:429-434. [PubMed: 11760618] 
Dantzer R, O'Connor JC, Freund GG, Johnson RW, Kelley KW. From inflammation to sickness and depression: when the immune system subjugates the brain. Nat. Rev. Neurosci. 2008; 9:46-56. [PubMed: 18073775]

Jylhä P, Isometsä E. The relationship of neuroticism and extraversion to symptoms of anxiety and depression in the general population. Depress. Anxiety. 2006; 23:281-289. [PubMed: 16688731]

Kemeny ME. The psychobiology of stress. Curr. Dir. Psychol. Sci. 2003; 12:124-129.

Kessler RC, Berglund P, Demler O, Jin R, Merikangas KR, Walters EE. Lifetime prevalence and ageof-onset distributions of DSM-IV disorders in the national comorbidity survey replication. Arch. Gen. Psychiatry. 2005; 62:593-602. [PubMed: 15939837]

Kiecolt-Glaser JK, McGuire L, Robles TF, Glaser R. Emotions, morbidity and mortality: new perspectives from psychoneuroimmunology. Annu. Rev. Psychol. 2002; 53:83-107. [PubMed: 11752480]

Kiecolt-Glaser JK, Preacher KJ, MacCallum RC, Atkinson C, Malarkey WB, Glaser R. Chronic stress and age-related increases in the proinflammatory cytokine IL-6. Proc. Natl. Acad. Sci. USA. 2003; 100:9090-9095. [PubMed: 12840146]

Kubzansky LD, Kawachi I. Going to the heart of the matter: do negative emotions cause coronary heart disease? J. Psychosom. Res. 2000; 48:323-337. [PubMed: 10880655]

Lieb K, Engelbrecht MA, Gut O, Fiebich BL, Bauer J, Janssen G, Schaefer M. Cognitive impairment in patients with chronic hepatitis treated with interferon alpha (IFN-alpha): results from a prospective study. Eur. Psychiatry. 2006; 21:204-210. [PubMed: 16632167]

Maes M, Song C, Lin A, De Jongh R, Van Gastel A, Kenis G, Bosmans E, De Meester I, Benoy I, Neels H, Demedts P, Janca A, Scharpé S, Smith RS. The effects of psychosocial stress on humans: increased production of pro-inflammatory cytokines and Th1-like response in stress-induced anxiety. Cytokine. 1998; 10:313-318. [PubMed: 9617578]

Miller GE, Chen E, Zhou ES. If it goes up, must It come down? Chronic stress and the hypothalamicpituitary-adrenocortical axis in humans. Psychol. Bull. 2007; 133:25-45. [PubMed: 17201569]

Mogg K, Bradley BP. Attentional bias in generalized anxiety disorder versus depressive disorder. Cogn. Ther. Res. 2005; 29:29-45.

Moons WG, Eisenberger NI, Taylor SE. Anger and fear responses to stress have different biological profiles. Brain Behav. Immun. 2010; 24:215-219. [PubMed: 19732822]

Pitsavos C, Panagiotakos DB, Papageorgiou C, Tsetsekou E, Soldatos C, Stefanadis C. Anxiety in relation to inflammation and coagulation markers, among healthy adults: the ATTICA Study. Atherosclerosis. 2006; 185:320-326. [PubMed: 16005881]

Ridker PM. High-sensitivity C-reactive protein: potential adjunct for global risk assessment in the primary prevention of cardiovascular disease. Circulation. 2001; 103:1813-1818. [PubMed: 11282915]

Roy-Byrne PP, Davidson KW, Kessler RC, Asmundson GJG, Goodwin RD, Kubzansky L, Lydiard RB, Massie MJ, Katon W, Laden SK, Stein MB. Anxiety disorders and comorbid medical illness. Focus. 2008; 6:467-485.

Selye, H. The Stress of Life. McGraw-Hill, New York: 1956.

Slavich, GM.; O'Donovan, A.; Epel, ES.; Kemeny, ME. Neurosci. Biobehav. Rev. Black sheep get the blues: a psychobiological model of social rejection and depression.

Thyer BA, Parrish RT, Curtis GC, Nesse RM, Cameron OG. Ages of onset of DSM-III anxiety disorders. Compr. Psychiatry. 1985; 26:113-122. [PubMed: 3987242]

Toker S, Shirom A, Shapira I, Berliner S, Melamed S. The association between burnout, depression, anxiety, and inflammation biomarkers: C-reactive protein and fibrinogen in men and women. J. Occup. Health Psychol. 2005; 10:344-362. [PubMed: 16248685]

Whooley MA, Caska CM, Hendrickson BE, Rourke MA, Ho J, Ali S. Depression and inflammation in patients with coronary heart disease: findings from the heart and soul study. Biol. Psychiatry. 2007; 62:314-320. [PubMed: 17434456]

Zhou D, Kusnecov AW, Shurin MR, DePaoli M, Rabin BS. Exposure to physical and psychological stressors elevates plasma interleukin 6: relationship to the activation of hypothalamic-pituitaryadrenal axis. Endocrinology. 1993; 133:2523-2530. [PubMed: 8243274] 
Zigmond AS, Snaith RP. The Hospital Anxiety and Depression Scale. Acta Psychiatr. Scand. 1983; 67:361-370. [PubMed: 6880820] 

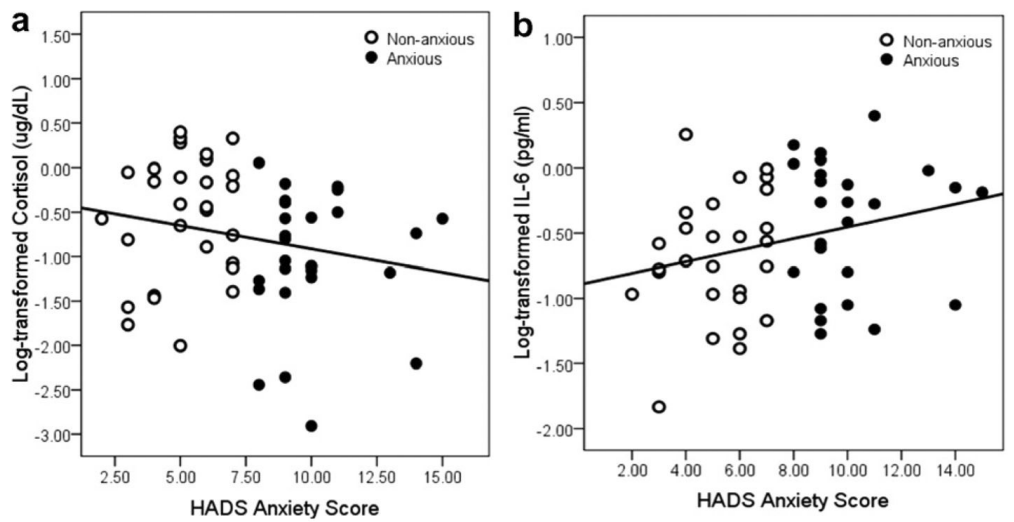

Fig. 1.

(a and b) Scatterplot illustrating between and within group differences in cortisol and IL-6. Correlational analyses indicated that increasing levels of anxiety tended to be associated with higher levels of IL-6 ( $r=.27, p=.05)$ and lower levels of cortisol $(r=-.25, p=.06)$. Furthermore, clinically anxious participants exhibited significantly lower levels of morning cortisol $(t=2.84, p=.006$ ) and significantly higher levels of interleukin-6 (IL-6; $t=2.01, p$ $=.05)$ than non-anxious participants. 


\section{Table 1}

Characteristics of clinically anxious and non-anxious participants.

\begin{tabular}{|c|c|c|c|}
\hline Variable & Anxious $(n=27)$ & Non-anxious $(n=29)$ & $P$ \\
\hline Age: $M(S D)$ & $33.52(8.04)$ & $30.97(10.21)$ & .30 \\
\hline Females: $n(\%)$ & $20(74.1)$ & $18(62.1)$ & .34 \\
\hline Fear of needles: $n(\%)$ & $6(22.2)$ & $6(20.7)$ & .89 \\
\hline Cold/Flu (past 3 months): $n(\%)$ & $8(29.6)$ & $10(34.5)$ & .70 \\
\hline Health complaints (past 3 months): $n(\%)$ & $7(25.9)$ & $9(31)$ & .67 \\
\hline OCP (females): $n(\%)$ & $16(80)$ & $11(61.1)$ & .20 \\
\hline \multicolumn{4}{|l|}{ Highest level of education: $n(\%)$} \\
\hline Primary & $1(3.7)$ & 0 & \\
\hline Secondary & $1(3.7)$ & 0 & \\
\hline Third level & $24(88.89)$ & $29(100)$ & .49 \\
\hline \multicolumn{4}{|l|}{ Marital status: $n(\%)$} \\
\hline Married & $5(18.5)$ & $6(20.7)$ & \\
\hline Living with partner & $5(18.5)$ & $6(20.7)$ & \\
\hline Separated/Divorced & $1(3.7)$ & 0 & \\
\hline Single & $16(59.3)$ & $17(58.6)$ & .96 \\
\hline \multicolumn{4}{|l|}{ Self-rated physical health: $n(\%)$} \\
\hline Excellent & $2(7.4)$ & $5(17.2)$ & \\
\hline Very good & $18(66.7)$ & $14(48.3)$ & \\
\hline Good & $5(18.5)$ & $10(34.5)$ & \\
\hline Fair & $1(3.7)$ & 0 & \\
\hline Poor & $1(3.7)$ & 0 & .89 \\
\hline Cortisol & $.44(.25)$ & $.70(.41)$ & .006 \\
\hline Interleukin-6 & $.72(.33)$ & $.56(.26)$ & .05 \\
\hline C-reactive protein & $1.14(1.77)$ & $1.06(1.90)$ & .88 \\
\hline
\end{tabular}

Notes: Group differences were calculated using $t$-tests, Chi-square tests and Mann Whitney $U$ tests as appropriate. $M=$ mean; $\mathrm{SD}=\operatorname{standard}$ deviation; $n=$ sample size; $\mathrm{OCP}=$ oral contraceptive pill. 